\title{
Cultura colaborativa e gestão do conhecimento em esporte e lazer
}

\author{
Rodrigo Duarte Ferrari \\ Giovani De Lorenzi Pires ${ }^{2}$
}

\author{
${ }^{1}$ Programa de Pós-Graduação em Educação da Universidade Federal de Santa Catarina, \\ Florianopolis, SC, Brasil \\ ${ }^{2}$ Departamento de Educação Física, Universidade Federal de Santa Catarina, Florianopolis, SC, Brasil
}

\begin{abstract}
Resumo: A partir da experiência de desenvolvimento e administração do RIRC, um projeto realizado pelo LaboMídia/UFSC com o apoio da Rede CEDES/Ministério do Esporte, delimitamos o uso desse repositório digital como objeto de pesquisa de uma dissertação de mestrado. Dessa forma, organizamos esse trabalho com base nas discussões teóricas que envolvem a gestão da informação e do conhecimento no âmbito do esporte e lazer brasileiro, sobretudo, pelos princípios do movimento mundial a favor da promoção do acesso aberto à informação e conhecimento científico. Nesse contexto, investigamos como o RIRC era percebido pelos gestores e pesquisadores da Rede CEDES, durante os anos de 2010 e 2011 . A delimitação deste problema de pesquisa criou condições para observar e refletir sobre o compromisso social e político dos integrantes da Rede CEDES em organizar e disponibilizar as produções dessa ação ministerial. Sendo assim, descrevemos, analisamos e refletimos sobre a percepção dos integrantes da Rede CEDES em relação ao RIRC, no escopo da gestão da informação e do conhecimento em esporte e lazer. Realizamos treze entrevistas com os integrantes da Rede CEDES no período de 19/11/2010 até 14/09/2011 e utilizamos algumas estatísticas do RIRC e da própria rede como dados. Por conta dessa estrutura, esta pesquisa se caracterizou como um estudo de caso qualitativo, entendimento que nos orientou durante a coleta, análise, discussão e interpretação dos dados. Após a construção dos caminhos teóricos e metodológicos definimos quatro eixos de discussão dos dados: a) Contexto sócio-histórico de construção da Rede CEDES; b) O processo de desenvolvimento do RIRC; c) O RIRC como política pública; e d) O RIRC no contexto da cultura participativa. O diálogo entre esses eixos e o referencial teórico fundamenta a apresentação dos achados desta pesquisa, dos quais destacamos inicialmente que o RIRC é percebido pelos integrantes da Rede CEDES entrevistados como uma iniciativa relevante no âmbito das políticas públicas em esporte e lazer no Brasil. Todavia, menos de $20 \%$ do potencial do sistema foi utilizado pelos integrantes da Rede CEDES no período em que realizamos essa investigação. Isso indica que há contradições entre um discurso pautado pelas qualidades ideais dos repositórios digitais e do acesso aberto em contraste com a prática efetiva que deveria materializar esse discurso. Entre outras reflexões, consideramos que o RIRC se configura como uma ferramenta que demonstra algumas necessidades relativas às possibilidades de mudanças culturais dos integrantes da Rede CEDES, no que se refere à produção e veiculação das informações e conhecimentos da rede, para que a proposta se efetive. Isso depende, principalmente, do Ministério do Esporte considerar a inclusão da gestão das informações e dos conhecimentos de interesse público em acesso aberto, como prioridade da política ministerial no campo do esporte. Com base na literatura científica e nos dados dessa pesquisa, compreendemos que a definição e o cumprimento de um mandado que garanta o autoarquivamento no RIRC pelos pesquisadores da Rede CEDES poderia ser um primeiro passo. Todavia, o RIRC é uma proposta isolada na estrutura do ME e não foi desenvolvido para atender todas as demandas desse ministério. Esse fato subsidia nossa compreensão de que não podemos pensar na materialização de uma proposta de gestão da informação e do conhecimento em esporte e lazer, como política pública do ME, enquanto o RIRC for a única ação concreta com esse propósito no ministério.
\end{abstract}

Palavras-chave: Esporte e lazer. Gestão da informação e do conhecimento. Acesso aberto. RIRC, Autoarquivamento.

\section{Collaborative culture and knowledge management in sport and leisure}

\begin{abstract}
From the development and administration experiences of RIRC, a project undertaken by LaboMídia/UFSC with the support of CEDES Network/Ministry of Sports, we delimited the use of this digital repository as an object for research of one master's degree dissertation. Thus, we organize this work based on theoretical discussions that involve the information and the knowledge management in the fields of sport and leisure in Brazil, especially, with the principles of the worldwide movement that emphasizes the promotion of open access to scientific information and knowledge. In this context, we investigated how the RIRC was perceived by managers and researchers of the CEDES Network during the first year of the system's operation. The delimitation of this research's problem, created conditions to observe and to ponder over the social and political commitment of the CEDES Network's members, to organize and to make the results of this action accessible. Therefore, we describe, analyze and reflect on the perception of this CEDES Network members in relation to RIRC, in the scope of the information and knowledge management in sport and leisure. We conducted interviews with thirteen members of the CEDES Network and used some statistics of the RIRC and from CEDES Network as data. Because of this structure, this research is characterized as a qualitative case study, this understanding guided us during the gathering, analysis, discussion and interpretation of data. After the construction of the theoretical and methodological structure, we defined four axes of discussions: a) socio-historical context of the building process of the CEDES Network; b) The development process of the RIRC; c) The RIRC as public policy; and d) The RIRC in context of participatory culture. The dialogue between these axes and the theoretical framework legitimize the purpose behind the presentation of the research's findings, which initially highlight the fact that RIRC is perceived by the CEDES members as an important initiative in the framework of public policies in sport and leisure in Brazil. However, less than $20 \%$ of the system's full potential was used by the CEDES Network during the period which we perform this research. This indicates that there are profound contradictions between the discourses guided by the ideal qualities of digital repositories and open access, in contrast to the effective practice that should materialize these discourses. Among other considerations, we believe that RIRC is configured as a tool that demonstrates the need to change the culture of the CEDES members, in regards to the production and dissemination of information and knowledge, in order to ensure that the proposal will come about appropriately. This depends mainly on the ME to consider, as a priority, enabling open access of all the information and knowledge of public interest and integrate that into the ministerial policies in the field of sport. Based on scientific literature and on the data from this study, we understand that the fulfillment of a mandate that ensures the self-archiving in RIRC by the researchers of CEDES Network could be a first step. However, the RIRC is an isolated proposal at ME's structure and was not designed to meet all demands of this ministry. This fact supports our understanding that as long as the RIRC is the only solid action in ministry, we can not consider the materialization of the sports' management scheme regarding information and knowledge, as a public policy of the ME.
\end{abstract}

Keywords: Sport and leisure. Information and knowledge management. Open access. RIRC. Self-archiving 


\section{Introdução}

De acordo com o artigo $27^{\circ}$ da Declaração Universal dos Direitos Humanos, "toda pessoa têm o direito de (...) participar do processo científico e de seus benefícios" (NACÕES

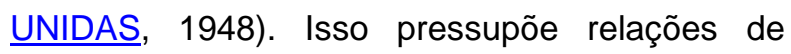
direitos e deveres entre a sociedade e diferentes atores do processo de produção do conhecimento científico. No caso deste texto, essa relação é observada a partir de três perspectivas no contexto da Rede de Centros de Desenvolvimento do Esporte Recreativo e do Lazer do Ministério do Esporte (Rede CEDES) e do seu Repositório Institucional da Rede CEDES (RIRC):

a) a dos direitos de cada cidadão brasileiro em ter acesso ao conhecimento científico produzido no campo do esporte e lazer, sobretudo pesquisadores e gestores públicos da área;

b) a dos deveres do Ministério do Esporte (ME) de organizar, preservar e disponibilizar as informações e conhecimentos científicos em esporte e lazer, haja vista que essa instituição financia pesquisas nesse campo;

c) a dos deveres de pesquisadores deste campo que recebem dinheiro público para produzir conhecimento científico no Brasil.

A Rede CEDES é uma ação de política pública implementada por convênios entre o ME e universidades distribuídas pelas cinco regiões do Brasil, estrutura que desde 2003 resultou no apoio à produção de diversas pesquisas no campo do esporte e do lazer brasileiro. Essas produções geraram demandas administrativas, sociais e políticas que se situam no âmbito da gestão da informação e do conhecimento científico. Dessa forma, os gestores da Rede CEDES identificaram que era preciso organizar, preservar e disponibilizar em acesso aberto (HUNGRIA, 2002) as produções científicas da rede, sobretudo, para auxiliar a construção e gestão de políticas públicas.

Essa demanda foi reconhecida pelo LaboMidia/UFSC (http://labomidia.ufsc.br/), parceiro do $\mathrm{ME}$, que propôs a criação de um repositório digital dessas pesquisas, ideia acolhida pelos demais pesquisadores da Rede CEDES, que aprovaram a criação dessa ferramenta colaborativa. Foi dessa forma que, em 2009, num projeto de parceria entre ME e LaboMidia/UFSC, surgiu e se consolidou o projeto de desenvolvimento do RIRC, uma ferramenta criada especificamente para reunir, organizar, preservar e disponibilizar as produções científicas da Rede CEDES.
O RIRC é uma iniciativa inovadora, tanto do ponto de vista técnico quanto da perspectiva política da gestão da informação e do conhecimento no Brasil, sobretudo em esporte e lazer. Avaliamos que esse contexto forma um fértil campo de investigação e, por isso, tomamos - RIRC como o objeto de estudo de uma pesquisa (FERRARI, 2012) que foi apresentada em 2012 como dissertação de mestrado ao Programa de Pós-Graduação em Educação Física da Universidade Federal de Santa Catarina (PPGEF/UFSC), tendo como problema de investigação: como a proposta de desenvolvimento do RIRC é percebida pelos gestores e pesquisadores da Rede CEDES, em relação ao compromisso social e político de tornar disponíveis, de forma organizada e permanente, as informações e conhecimentos produzidos pela rede? (FERRARI, p. 36, 2012)

Como consequência deste questionamento, descrevemos, analisamos e refletimos sobre a percepção dos integrantes da Rede CEDES relativa ao RIRC, considerando as características, propósitos e funcionalidades da ferramenta para gerir informações e conhecimentos. Sendo assim, pretendemos com este texto apresentar as principais discussões e resultados desta pesquisa.

Do ponto de vista metodológico esta pesquisa se caracterizou como um estudo de caso (BOGDAN; BINKLEN, 1991) que tomou o RIRC como seu objeto de análise. A técnica de pesquisa que utilizamos foi a observação participante, escolha que nos permitiu estudar 0 RIRC e a comunidade da Rede CEDES a partir e "por dentro" dela.

O instrumento principal de produção dos dados foram 13 (treze) entrevistas semiestruturadas, realizadas com os gestores do ME e coordenadores de núcleos e projetos de pesquisas da Rede CEDES. As entrevistas aconteceram entre novembro/2010 e setembro/2011. O critério de seleção das gestoras foi o vínculo direto com a administração da Rede CEDES. Os pesquisadores foram selecionados de acordo com a relevância e histórico de participação na rede, assim como a localização geográfica, para abranger quatro regiões do país, excetuando a região norte por não dispor de pesquisas concluídas na ocasião em que os dados foram coletados. Nove entrevistas foram realizadas presencialmente, três 
via videoconferência e uma por troca de correspondência eletrônica (email). Em média cada entrevista durou uma hora, todas foram gravadas em formato audiovisual, transcritas e enviadas para os pesquisadores aprovarem o conteúdo, sendo autorizada a identificação de seus nomes. Diante deste quadro metodológico, o desenho investigativo dessa pesquisa se caracteriza predominantemente como um estudo qualitativo (GOELLNER et al, 2010).

Também trabalhamos com descrições analíticas e dados quantitativos da Rede CEDES e do RIRC. Esses dados foram coletados a partir da data de lançamento oficial do RIRC, 19 de junho de 2010, até 14 de setembro de 2011, pois essa foi a data da última entrevista realizada e marcou o fim da fase de coleta de dados. Os dados quantitativos e qualitativos foram triangulados e apresentados a partir de eixos de discussões.

$\mathrm{Na}$ análise inicial do material de campo, definimos quatro eixos temáticos de discussão, ou seja, temas recorrentes e com características semelhantes que puderam ser organizados como unidades de registro definidas a partir do conteúdo das entrevistas realizadas. São eles: a) Contexto sócio-histórico de construção da Rede CEDES e do RIRC; b) O processo de desenvolvimento do RIRC; c) O RIRC como política pública; d) O RIRC no contexto da cultura participativa.

Esses eixos temáticos foram criados com base nos critérios que Bardin (2009) denomina como exclusão mútua, ou seja, definição de uma identidade clara e homogênea de cada eixo. Num segundo momento, os eixos definidos orientaram o estudo e a definição de referenciais teóricos capazes de fundamentar as discussões que propomos nessa pesquisa, assim como a interpretação dos dados de acordo com nosso problema, objetivos e questões de investigação deste trabalho. De acordo com essas considerações introdutórias, apresentaremos a seguir uma síntese das discussões dos eixos definidos na pesquisa, assim como nossas conclusões.

\section{O RIRC em Discussão}

Nos dois primeiros eixos de discussão, contexto sócio-histórico de construção da Rede CEDES e do RIRC e o processo de desenvolvimento do RIRC, os entrevistados relataram que a posse do presidente Luiz Inácio Lula da Silva é um dos marcos que define o início de mudanças significativas no âmbito do esporte brasileiro. A criação do ME pelo presidente, em janeiro de 2003, se configura como um dos atos fundadores dessas transformações.

Inicialmente, o ME foi organizado em quatro secretarias: Executiva; Alto Rendimento; Esporte Educacional e a Secretaria Nacional de Desenvolvimento de Esporte e Lazer (SNDEL), espaço onde a Rede CEDES foi criada com o principal objetivo de compensar os investimentos em pesquisas realizadas com vistas ao esporte de alto rendimento.

a Rede CEDES surge para suprir uma
necessidade de produção do conhecimento no
âmbito do desenvolvimento do esporte e lazer
de cunho social, contrapondo-se a Rede
CENESP, que é voltada para o esporte de alto
rendimento (Dra. Ana Márcia, entrevista
realizada 20/06/2011).

De acordo com informações disponibilizadas no site do $\mathrm{ME}$, a construção histórica da Rede CEDES é dividida em quatro momentos. Os dois primeiros caracterizam-se pelo estabelecimento de parcerias e da consequente ampliação das mesmas, com grupos de pesquisas e universidades com méritos reconhecidos pelos dirigentes da SNDEL. O terceiro momento é definido pela criação do primeiro edital público. Por fim,

(...) o quarto momento da Rede é marcado pela ampliação do fomento à pesquisa social sobre Políticas Públicas de Esporte e Lazer e pelo investimento na gestão do conhecimento produzido pela Rede CEDES, sua sistematização e socialização ampla, buscando contribuir com a qualificação das demais ações do Programa Esporte e Lazer na Cidade (PELC) e a capacitação de gestores, agentes e estudiosos de esporte e lazer brasileiros e de outros países (MINISTÉRIO DO ESPORTE, 2011).

É no quarto momento que surge a ideia de desenvolvimento do RIRC, principalmente como estratégia para sistematizar e socializar a produção da rede. Também é possível observar uma fase de transição para o quinto momento da Rede CEDES, em meio a uma atmosfera de incertezas e especulações marcadas pela extinção oficial da SNDEL.

No período de 2003 a 2008, a Rede CEDES financiou um total de 208 pesquisas (KAWAGUTI, 2010). A autora também apresenta dados referentes à distribuição das pesquisas no Brasil, que apesar de atingirem as cinco regiões do país, 
favorecem mais as regiões Sul e Sudeste que concentram a maior parte das mesmas. Essa realidade representa as diferenças econômicas e sociais brasileiras, assim como o desafio de equilibrar essa situação com as políticas públicas em esporte e lazer. A pesquisadora ilustra os eixos temáticos da Rede CEDES, assim como a porcentagem de pesquisas em cada tema.

Tabela 1. Distribuição das pesquisas nos nove eixos temáticos, representados em porcentagem (KAWAGUTI, p. 55, 2010).

\begin{tabular}{|l|c|}
\hline \multicolumn{1}{|c|}{ EIXO TEMÁTICO } & $\%$ \\
\hline Memória do esporte e lazer & 19,58 \\
\hline Perfil do esporte e lazer & 8,47 \\
\hline Programas integrados de esporte e lazer & 15,34 \\
\hline Desenvolvimento de programas sociais de esporte e lazer & 8,99 \\
\hline Observatório do esporte & 10,58 \\
\hline Gestão de esporte e lazer & 13,23 \\
\hline Avaliação de políticas e programas de esporte e lazer & 6,35 \\
\hline Infraestrutura de esporte e lazer & 16,40 \\
\hline Sistema nacional de esporte e lazer & 1,06 \\
\hline TOTAL & $\mathbf{1 0 0}$ \\
\hline
\end{tabular}

Chama a atenção a maior porcentagem de pesquisas históricas que procuram resgatar e documentar o passado esportivo e o lazer brasileiro. Esses dados fortalecem diretamente a ideia do RIRC, no sentido de organizar e preservar a memória para compreender 0 presente e planejar o futuro das políticas públicas no país. Em congruência com esse trabalho, Tavares (2010) descreve e quantifica os produtos das pesquisas financiadas pela Rede CEDES.

Tabela 2. Quantidade total de produtos da Rede CEDES no período de 2003/2008.

\begin{tabular}{|l|c|}
\hline \multicolumn{1}{|c|}{ Produtos Rede CEDES - 2003/2008 } & $\mathbf{N}^{\circ}$ \\
\hline Artigos (anais e periódicos) & 226 \\
\hline Resumos & 317 \\
\hline Capítulos de livros & 80 \\
\hline Home Page & 26 \\
\hline Organização de eventos & 53 \\
\hline Palestras & 49 \\
\hline Outros livros & 8 \\
\hline Apresentação de trabalhos & 163 \\
\hline Vídeos/Entrevistas & 41 \\
\hline Monografias, dissertações e teses & 32 \\
\hline TOTAL & 995 \\
\hline
\end{tabular}

De acordo com os entrevistados, esses 995 documentos, de informações e conhecimentos sistematizados na área do esporte e lazer, contribui potencialmente com o desenvolvimento da política pública nacional. Porém, para isso é preciso que eles estejam organizados e disponíveis, principalmente para o acesso dos gestores dessa área, que estão envolvidos com o Programa Esporte e Lazer na Cidade (PELC). Rede CEDES e PELC são iniciativas interdependentes, em que os conhecimentos produzidos pela primeira devem contribuir com as demandas da segunda. Todas as produções listadas na tabela 2 são digitalizáveis e estão distribuídas pelas cinco regiões brasileiras; isto, além de justificar e atribuir sentido ao desenvolvimento do RIRC, legitima o status do sistema como uma eficiente ferramenta para aproximar a pesquisa científica e a sociedade no âmbito do esporte e lazer.

Porém, a secretária da SNDEL observa que um dos principais limites operacionais do RIRC é o reduzido número de recursos humanos, financeiros e de infraestrutura da própria SNDEL. A consequência dessa situação é apresentada a partir do seguinte depoimento: "Ao fazer 0 primeiro levantamento de relatórios eu tive que buscar em processos que estavam perdidos pelo $M E$, encontrei processos de até 6 anos que estavam no arquivo morto (Coordenadora/DCTEC/SNDEL - Patrícia Z. M. de Moraes, entrevista realizada 19/11/2010). Nesse sentido, desde junho de 2010, o RIRC se configura como uma ferramenta para solucionar esse tipo de problemática, contudo é apenas uma ação isolada frente as demandas de todo ME.

A pesquisa de Schwartz (2010) mapeou 126 pesquisas concluídas ou em andamento, sendo que apenas 28 extratos de relatórios estão disponíveis no RIRC, o equivalente a $22 \%$ do total. Com relação aos produtos (livros, capítulos de livros, artigos publicados em periódicos e congressos, fotos, etc) decorrentes de cada pesquisa foram mapeados 995 itens que poderiam estar disponibilizados nas coleções Produções de cada sub-comunidade do repositório, no entanto, apenas 149 estavam disponíveis no RIRC, equivalente a $15 \%$ do que foi mapeado na pesquisa. Esses dados representam um ponto central para enfrentar o problema desta pesquisa e fundamenta no plano quantitativo a observação de que o RIRC não foi apropriado pela comunidade da Rede CEDES de forma satisfatória na primeira fase de povoamento (processo de construção do acervo do RIRC) do sistema.

No eixo de discussão o RIRC como política pública, destacamos a diferença de investimentos e prioridades entre os programas do ME voltados para a educação e lazer. De acordo com as gestoras da SNDEL, o orçamento total da Rede CEDES previsto para o edital de 2011 foi de $\mathrm{R} \$ 1$ milhão, um montante muito baixo, por exemplo, em comparação com o Programa Segundo Tempo (PST, outro programa do ME), que 
recebeu mais de $\mathrm{R} \$ 255$ milhões no mesmo ano. Como consequência deste contexto, um dos pesquisadores entrevistados relata:

Vivemos uma total insegurança com relação as fontes de financiamento. As cifras, um milhão a cada ano, que se repetiu durante quase todo segundo mandato, é um recurso muito baixo em relação a outras políticas e programas (Dr. Fernando Mascarenhas, entrevista realizada 25/05/2011).

A despeito dos méritos de cada ação ministerial, segundo a secretária da SNDEL essa diferença orçamentária se reflete objetivamente nas probabilidades de consolidação da Rede CEDES. Isso ocorre, principalmente, porque as políticas públicas são avaliadas por técnicos em economia e o primeiro critério de análise para medir o impacto e importância dessas políticas é a verba alocada em cada programa ou ação. Segundo Aragão (1992), esse cenário exemplifica o que o sociólogo alemão Jürgen Habermas identifica como a invasão da dimensão sistêmica da sociedade com relação ao mundo da vida. As políticas públicas e a administração do estado obedecem a uma razão monológica, instrumental, que supervaloriza um tipo de cálculo matemático e procedimentos burocráticos para ler o presente e planejar o futuro.

Você está dentro de uma estrutura disputando espaços com outros, então se fala que existe pouca verba e prioridades. Isso acaba que, você se vê quase que legislando em causa própria e buscando recursos para tua secretaria. (Secretária/SNDEL - Rejane Penna Rodrigues, entrevista realizada 19/11/2010).

Por outro lado, de acordo com a pesquisadora Dra. Dulce Suassuna a construção das políticas públicas em esporte e lazer (PELC e Rede CEDES) no Brasil são pioneiras e deveriam ser um exemplo a ser seguido por todo governo federal. $O$ fundamento desse diagnóstico reside na estratégia de articular o PELC com a Rede CEDES, no sentido de trabalhar com a produção de conhecimento científico para subsidiar as políticas públicas da área, materializando um exercício contínuo de construção e autoavaliação.

A Rede CEDES forma um canal de diálogo crítico entre o poder público e a sociedade, aumentando o valor da dimensão simbólica inerente às políticas públicas, que na nossa compreensão, devem ser pensadas e colocadas em prática a partir das complexas necessidades que partem da sociedade para o governo, e não o inverso. Outro dado relevante que surgiu nesta pesquisa se refere ao Centro de Documentação e
Informação do Ministério do Esporte (CEDIME), sobretudo, por sua existência oficial como um programa desta instituição e sua inexistência de fato. O depoimento da secretaria da SNDEL confirma essa realidade.

Estou a 4,5 anos tentando entender o CEDIME. Para mim uma das partes do CEDIME seria ter um repositório. Porém vejo o CEDIME como uma sigla que não diz nada para dentro e fora do ME, a não ser para os especialistas (Secretária/SNDEL - Rejane Penna Rodrigues, entrevista realizada 19/11/2010).

O caso do CEDIME ilustra que não há de fato uma proposta objetiva de gestão da informação e do conhecimento como parte da Política Nacional do Esporte, apesar de contraditoriamente serem contempladas na primeira ação e meta da III Conferência Nacional do Esporte. O CEDIME é um programa de interesse público e expressa a falta de prioridade e atenção que a gestão da informação e do conhecimento recebe por parte dos gestores do ME.

No âmbito político, o RIRC foi concebido a partir dos fundamentos do movimento mundial a favor do acesso aberto (HUNGRIA, 2002; COSTA, 2006) e dos softwares livres (DIBONA, 1999; BRANCO, 2004). Harnad (2011) complementa que $\mathrm{o}$ acesso aberto também é uma questão jurídica e precisa ser legalmente normatizado para que não dependa apenas da vontade política de alguns gestores públicos que se alinham com a proposta. Compreendemos que esses princípios deveriam ser a base para uma futura proposta de gestão da informação e do conhecimento relativa a todo ME

Em meio a estas considerações, todos os entrevistados reconhecem o RIRC como uma importante ação da Rede CEDES, mas na prática esse discurso se torna contraditório, pois como observamos anteriormente, menos de $20 \%$ do potencial do RIRC está sendo aproveitado pelos pesquisadores da Rede CEDES. Em certa medida esses dados não representam uma novidade ou anomalia no âmbito do desenvolvimento de outros repositórios digitais. De acordo com Harnad (2011), os repositórios digitais não funcionam sem um mandado que normatize esse comportamento rigorosamente. Um repositório digital sem mandado possui em média uma porcentagem de autoarquivamento de $15 \%$.

O autoarquivamento é definido como o depósito efetuado pelos próprios pesquisadores 
de suas respectivas produções científicas em repositórios digitais de acesso aberto. Esse comportamento é caracterizado pelo compartilhamento de informações e conhecimentos científicos no formato digital, que surgem no ciberespaço.

Este é o ponto de transição que nos leva a discutir o RIRC num contexto colaborativo. $O$ RIRC é uma ferramenta que surge a partir das recentes possibilidades de transformações culturais vinculadas à expansão do ciberespaço, especialmente, a partir do conceito de inteligência coletiva (LÉVY, 2010), no sentido do surgimento de novas possibilidades de práticas colaborativas e trabalho em rede. Jenkins (2009) denomina esse contexto de potenciais mudanças de cultura participativa, sendo que os repositórios digitais e o autoarquivamento são mecanismos que operam nessa lógica.

No campo da pesquisa em esporte e lazer, as principais inovações do RIRC são conceituais, pois o uso técnico da ferramenta obedece à mesma lógica de sistemas já consolidados nesse campo acadêmico. Estamos nos referindo aos periódicos já adaptados ao Sistema Eletrônico de Editoração de Revistas (SEER) e aos trabalhos submetidos a congressos via Sistema On-line de Apoio a Congressos (SOAC), que possuem o processo comum de cadastro do usuário, preenchimento dos metadados e submissão dos arquivos digitais. Os principais periódicos da área utilizam a plataforma SEER e o principal congresso, CONBRACE, utiliza o SOAC desde 2008. Por outro lado, observamos que a proposta conceitual de autoarquivamento não mobilizou significativamente os pesquisadores da Rede CEDES a utilizarem o RIRC, ao contrário do que vêm ocorrendo com a utilização do SEER e SOAC.

Um dos pesquisadores da Rede CEDES nos fornece uma possibilidade para refletir sobre esse tema:

[...] o retorno para o pesquisador no que se refere à avaliação, principalmente dos periódicos, onde existe interesse (avaliação) dos pesquisadores em publicar nesses espaços, enquanto no repositório ganha menos o pesquisador e mais o campo (Dr. Fernando Mascarenhas, entrevista realizada 25/05/2011).

No campo acadêmico brasileiro publicar os resultados de pesquisas, sobretudo, em periódicos é uma questão de sobrevivência, especialmente dos profissionais que atuam em programas de pós-graduação. Portanto, se cadastrar em periódicos eletrônicos e utilizar essas ferramentas para submeter trabalhos envolve um alto grau de interesse pessoal por parte dos pesquisadores, pois a permanência e o prestígio desses profissionais na pós-graduação dependem desse comportamento. No caso dos repositórios digitais, esse interesse é mais fraco.

\section{[...] somente os professores que estão vinculados aos programas de pós-graduação já se colocaram em pauta a questão da divulgação do conhecimento e precisam fazer isso pela própria sobrevivência do sistema de pós graduação, mas fazemos isso mais enfaticamente naquilo que nos dá um resultado imediato, no Lattes (Ana Márcia, entrevista realizada 14/07/2011).}

Concordamos com a fala da pesquisadora, porém, segundo Leite (2009), os benefícios para os pesquisadores que auto-arquivam suas produções em repositório não podem ser desconsiderados. São eles: organização da informação e do conhecimento da rede de trabalho que eles participam; aumento da visibilidade e impacto de suas pesquisas; prestação de contas com a sociedade; ampliação das possibilidades de diálogo entre os pesquisadores da própria rede e com a sociedade em geral. Na mesma lógica argumentativa dos pesquisadores, a diretora do DCTEC reconhece que esses aspectos não têm sido suficientes para mobilizar os pesquisadores a assumirem a responsabilidade de construir o RIRC. Consideramos que esta problemática identificada é uma questão moral, o que nos mobiliza a convidar Habermas (1989) para nossas reflexões e definir um posicionamento filosófico a partir do qual podemos abordar a temática.

\begin{abstract}
Discutimos questões práticas do tipo: "O que devo/devemos fazer?", na pressuposição de que a resposta não possa ser qualquer uma; nós nos acreditamos capazes em princípio de distinguir as normas e mandamentos corretos dos falsos. Se, por um lado, as proposições normativas não são passíveis de verdade em sentido estrito, por conseguinte não no mesmo sentido em que os enunciados descritivos podem ser verdadeiros ou falsos, temos que colocar o problema de explicar o sentido de "verdade moral" (...) (HABERMAS, 1989, p. 77).
\end{abstract}

Nesse caso, a "verdade moral" inerente ao uso do RIRC como um dever dos pesquisadores da Rede CEDES se fundamenta no enunciado de que o RIRC é um projeto de interesse público e que o autoarquivamento é um compromisso social e político dos pesquisadores que são financiados com recursos públicos. Se os 
pesquisadores concordarem que suas profissões pertencem a um projeto coletivo orientado pela construção democrática do nosso país, eles não precisam de mais nenhum motivo, além deste, para autoarquivar suas produções no RIRC.

Entretanto, essa tensão entre interesses públicos e privados extrapola as fronteiras do RIRC e se configura como um desafio que permeia a estrutura da Rede CEDES como um todo. De acordo com os pesquisadores e gestores da Rede CEDES, a própria organização do trabalho em rede ainda é um processo que está em construção, pois "não há muita tradição na produção em rede ainda" (Dr. Giuliano Pimentel, entrevista realizada 30/06/2011) no campo do esporte e lazer brasileiro. Pra Castells (2010) a organização em rede é o novo paradigma da organização econômica que começa a se estruturar na década de $1980 \mathrm{com}$ a transição dos modelos industriais fordistas para o toyotismo. Essa estrutura prevê o aumento da eficiência no campo do trabalho por meio do investimento em ações colaborativas, flexibilidade e descentralização de cargos e funções, maior autonomia nos processos de tomada de decisões e do investimento para facilitar o fluxo das informações, comunicações e conhecimentos. Porém, ao mesmo tempo em que essa proposta aumenta a eficiência da organização e resultados do trabalho, ela é mais difícil de ser estruturada. Segundo a Secretária da SNDEL,

Essa vivência democrática é difícil de ser aceita, porque dependendo do histórico de algumas instituições ou pessoas, é melhor ter alguém para obedecer ou repudiar. Portanto, na forma como a rede é estruturada, cada um deve assumir sua parte de responsabilidade (Secretária/SNDEL - Rejane Penna Rodrigues, entrevista realizada 19/11/2010).

A gestora traz para o diálogo um aspecto central para o funcionamento da organização do trabalho em rede, o aumento da responsabilidade de cada integrante da rede e uma proposta mais horizontal, coletiva e colaborativa de trabalho. No caso especifico do RIRC, isso é representado na distribuição coletiva da responsabilidade em relação à construção da ferramenta, pois seguindo a proposta original vinculada com os repositórios digitais (HARNAD, 2011), sem o autoarquivamento dos pesquisadores o RIRC não funciona.

Nesse sentido, o entendimento mútuo (HABERMAS, 1989) sobre a legitimidade dos deveres morais dos pesquisadores da Rede
CEDES em relação ao RIRC e a proposta de trabalho em rede descrita por Castells (2010) estabelecem os fundamentos para abordar a temática na perspectiva do direito positivo. Com isso, as normas moralmente aceitas e validadas pela comunidade da Rede CEDES devem ser sistematizadas na forma de um contrato objetivo e claro que defina as obrigações de cada integrante da rede, assim como as consequências caso esse acordo nãos seja cumprido. Isso representa a necessidade de elaboração de um mandado que garanta 0 autoarquivamento no RIRC de acordo com o inciso 37 do $5^{\circ}$ artigo da Constituição da República Federativa do Brasil de 1988. Essa proposta pode ser pensada a partir dos editais da Rede CEDES de 2009 e 2011, respectivamente, que são as únicas referências normativas às obrigações dos pesquisadores com o RIRC:

13.5. Os convênios firmados deverão apresentar como produtos:

a) relatório impresso da pesquisa, digitalizado e indexado no Repositório da Rede CEDES, pelo coordenador de pesquisa, após parecer da avaliação da prestação de contas (MINISTÉRIO DO ESPORTE, 2009, sem grifo no original).

14.5. Os convênios firmados deverão apresentar como produto final:

a) relatório impresso da pesquisa, digitalizado e indexado no Repositório da Rede CEDES, pelo coordenador de pesquisa, após parecer da avaliação da prestação de contas (MINISTÉRIO DO ESPORTE, 2011, sem grifo no original).

De acordo com o item 13.5. do edital de 2009, entendemos que 0 autoarquivamento dos relatórios de cada pesquisa financiada pela Rede CEDES configurava-se como um mandado, isto é, era um dever de cada coordenador de pesquisa. Nesse caso, a principal falha desse documento é não objetivar o que acontece ao responsável pelas pesquisas se $o$ autoarquivamento não fosse realizado. A proposta defendida pelo LaboMídia, no que se refere à entrada do autoarquivamento/RIRC no edital da Rede CEDES de 2009, era que esse comportamento fosse, além de um dos critérios de cumprimento do objeto, condição para participar nos próximos processos de concorrência (editais) da Rede CEDES.

Todavia, isso não ocorreu em 2009 nem em 2011, pois novamente esse conteúdo não está presente no documento que estabelece as regras para concorrer ao financiamento da Rede CEDES de 2011. Por exemplo, os coordenadores de pesquisa contemplados com financiamento pelo edital de 2009 e que não procederam ao 
autoarquivamento de suas produções puderam submeter novamente propostas ao edital de 2011 e serem outra vez contemplados, já que este não pressuponha o atendimento ao previsto no edital de 2009 como critério de submissão nem de avaliação de novas propostas. Isso enfraquece a Rede CEDES e principalmente o RIRC, que dificilmente será respeitado como um dever legítimo dos coordenadores de pesquisas financiadas pelo $\mathrm{ME}$, se ele é apenas citado de forma marginal no edital de chamada pública dessa ação ministerial, sem maiores consequências.

Diante desse quadro, concluímos que ainda não existe uma proposta de mandado capaz de garantir o autoarquivamento e o funcionamento adequado do RIRC pelo caminho do direito positivo. Isso nos impõe o desafio, por enquanto, de pensar a construção do RIRC no âmbito da consciência moral dos pesquisadores e se eles não desejarem utilizar a ferramenta, nada pode ser feito. Inclusive, a ausência de um mandado abre brechas para questionamentos que descaracterizam o próprio RIRC como repositório digital, uma ferramenta constitutivamente colaborativa que foi desenvolvida para funcionar por meio do autoarquivamento (HARNAD, 2004).

Nesse cenário, a autonomia, o compartilhamento e a colaboração são características centrais nos processos de comunicação e socialização das informações e conhecimentos. Dessa forma, ressaltamos novamente que estamos nos referindo ao RIRC como uma experiência concreta de distribuição cognitiva e inteligência coletiva (LÉVY, 2010) que ocorre em torno das relações que se estabelecem na formação de uma cultura participativa (JENKINS, 2009).

No âmbito destas discussões sobre o RIRC, já é possível observar alguns desdobramentos desta experiência. Em apenas um ano de funcionamento, o RIRC foi tomado como modelo para o desenvolvimento de outro repositório digital no campo da educação física e esportes, que é coordenado pela professora da Universidade Federal do Rio Grande do Sul (UFRGS), Dra. Silvana Goellner, que também faz parte da Rede CEDES.

Eu não conhecia os repositórios, conheci a ferramenta através do RIRC. Um dos grandes desejos que eu sempre tive em relação ao Centro de Memória do Esporte da UFRGS CEME era de colocar esse acervo que temos de forma digitalizada e disponível para quem quiser acessar (Dra. Silvana Goellner, entrevista realizada 13/09/2011).

Além do vínculo com a Rede CEDES, a pesquisadora também é responsável pelo projeto de organização, preservação e disponibilização da memória do PST, ligado ao repositório digital Centro de Memória do Esporte - CEME. Com base nos exemplos do RIRC e dos repositórios do CEME e do PST, lembramos que os repositórios digitais foram concebidos para funcionar sob a lógica do autoarquivamento, conforme Harnard (2004). Compreendemos que esse comportamento é a possibilidade mais inovadora e positiva inerente a essas ferramentas. $O$ fundamento desse entendimento reside, sobretudo, nas reflexões de Lévy (2010) e Jenkins (2009), por meio dos conceitos de cibercultura, inteligência coletiva e cultura participativa. As contribuições de Habermas (ARAGÃO, 1992), também são centrais para fundamentar o autoarquivamento como uma proposta democrática e potencialmente transformadora, pois ele representa um caminho para a autonomia. Por conta disso, compreendemos que o RIRC se configura como uma proposta única de gestão da informação e do conhecimento no campo do esporte e lazer brasileiro, quanto à valorização do princípio fundamental e operacional do autoarquivamento.

\section{Conclusão}

Observamos que o RIRC é percebido pelos integrantes da Rede CEDES, gestores e pesquisadores entrevistados, como uma iniciativa estratégica no caminho para a qualificação da política pública brasileira em esporte e lazer. Entendemos que apesar de algumas limitações, os entrevistados compreendem os propósitos básicos do sistema, tanto os conceituais quanto os práticos. Porém, esse contexto demonstra profundas contradições entre os discursos que enaltecem o RIRC e sua relevância acadêmica, política e social, e os poucos esforços coletivos dos integrantes da rede em relação à submissão dos produtos no RIRC, indicam que ele é subutilizado pelos pesquisadores, que usam menos de $20 \%$ da capacidade da ferramenta, e pelos gestores do ME, que não percebem o RIRC como uma prioridade da Rede CEDES e do ME. Segundo Harnad (2011), a elaboração e cumprimento de um mandado seria necessária para modificar esse cenário. 
Junto com o mandado, observamos que o entendimento da organização do trabalho em rede e da centralidade da informação e do conhecimento em nossa sociedade (CASTELLS, 2010); do papel do ciberespaço (LÉVY, 2010); e as possibilidades da construção de uma cultura participativa (JENKINS, 2009) são conceitos que poderiam contribuir com o fortalecimento do RIRC e do acesso aberto como política pública do ME. Entretanto, isso depende da reestruturação do complexo jogo de interesses que envolvem as decisões do poder público.

Por fim, reportamo-nos aos já referidos achados da pesquisa de Kawaguti (2010), dispostos na tabela 2, mostrando que os estudos do eixo memória do esporte e lazer da Rede CEDES representam quase um quinto das pesquisas $(19,58 \%)$, grande parte delas relacionadas a levantamento de fontes para construção de centros de memória. Diante desses dados, compreendemos que a melhor utilização do RIRC e a ampliação dos repositórios digitais facilitaria a realização de futuras pesquisas nessa perspectiva. Aproveitamos essa reflexão para finalizar este texto, realçando a relevância atual do autoarquivamento no âmbito da gestão da informação e do conhecimento com relação o nosso presente, até porque, no futuro, serão essas produções que possibilitarão as pesquisas sobre a memória do esporte e lazer no Brasil.

\section{Referências}

ARAGÃO, L. M. C. Razão comunicativa e teoria social crítica em Jürgen Habermas. Rio de Janeiro: Tempo Brasileiro, 1992.

BARDIN, L. Análise de Conteúdo, Edição revisada e atualizada. Lisboa: Edições 70, 2009.

BOGDAN, R.; BINKLEN, S. Investigação qualitativa em educação: uma introdução à teoria e métodos. Porto: Porto Editora, 1991.

\section{BRANCO, M. D. Software Livre na}

Administração Pública Brasileira, 2004.

Disponível em:

$<$ http://www.livrosgratis.com.br/arquivos livros/s10 00002.pdf $>$. Acesso em: 12 jun. 2011.

CASTELLS, M. A sociedade em rede. 6.ed. São Paulo: Paz e Terra, 2010.

COSTA, S. M. S. Filosofia aberta, modelos de negócios e agências de fomento: elementos essenciais a uma discussão sobre o acesso aberto à informação científica. Ciência da
Informação, Brasília, v. 35, n. 2, p. 39-50, maio/ago, 2006.

DIBONA, C. et al. Open sources: voices from the open source revolution. 1999. Disponível em:< http://oreilly.com/catalog/opensources/book/toc.ht ml>. Acesso em: 16 abril 2013

FERRARI, R. D. Gestão da informação e conhecimento em esporte e lazer: o caso do repositório institucional da rede CEDES (RIRC). 171 p. Dissertação (Mestrado em Educação Física). Universidade Federal de Santa Catarina, Florianópolis, 2012.

GOELLNER, S. et al. Pesquisa qualitativa na Educação Física brasileira: marco teórico e modos de usar. Revista da Educação

Física/UEM. Maringá, v.2, n. 3, p. 381-410, 2010.

HABERMAS, J. Consciência moral e agir comunicativo. Rio de Janeiro: Tempo Brasileiro, 1989.

HARNAD, S. et al. The green and the gold roads to Open Access. Nature Web Focus, 2004a.

HARNAD, S. Open Access to Research: Changing Researcher Behavior Through University and Funder Mandates. Journal of Democracy and Open Government. Reino Unido, v. 3, n. 1, p. 33-41, 2011.

HUNGRIA. Busapest Open Access Initiative. 2002. Disponível em:

$<$ http://www.soros.org/openaccess/read $>$. Acesso em 12 dez. 2011.

KAWAGUTI, C. N. Análise dos projetos financiados pela Rede CEDES. In: SCHWARTZ, G. M. (org.). Gestão da Informação sobre esporte recreativo e lazer: Balanço da Rede CEDES. Várzea Paulista: Fontoura, 2010.

JENKINS, H. Cultura da convergência. 2. ed. São Paulo: Aleph, 2009.

LEITE, F. C. L.. Como gerenciar e ampliar a visibilidade da informação científica brasileira: repositórios institucionais de acesso aberto. Brasília: IBICT/MCT, 2009.

LÉVY, P. Cibercultura. 3 ed. São Paulo: Editora 34, 2010.

MINISTÉRIO DO ESPORTE. Edital Rede CEDES. 2009. Arquivo pessoal, disponível em pdf.

MINISTÉRIO DO ESPORTE. Edital Rede

CEDES. 2011. Disponível em:

$<$ http://esporte.gov.br/arquivos/snelis/esporteLaze 
r/cedes/editalPelcRedeCedes2011.pdf.>. Acesso em: 16 abril 2013.

NAÇÕES UNIDAS. Declaração Universal dos Direitos Humanos. Adotada e proclamada pela resolução 217 A (III) da Assembléia Geral das Nações Unidas, 1948.

SCHWARTZ, G. M. (Org.). Gestão da Informação sobre esporte recreativo e lazer: Balanço da Rede CEDES. Várzea Paulista:

Fontoura, 2010.

TAVARES, G. H. Produtos e desdobramentos das pesquisas financiadas pela Rede CEDES. In: SCHWARTZ, G. M. (org.). Gestão da Informação sobre esporte recreativo e lazer: Balanço da Rede CEDES. Várzea Paulista: Fontoura 2010.

\section{Endereço:}

Rodrigo Duarte Ferrari

Rua Recanto das Gaivotas, 229 Rio Tavares

Florianópolis SC Brasil

88048-410

Telefone: (41) 9959-4409

e-mail: rd.ferrari@gmail.com

Recebido em: 07 de maio de 2012.

Aceito em: 19 de março de 2013.

\section{(c) (1)}

Motriz. Revista de Educação Física. UNESP, Rio Claro, SP, Brasil - elSSN: 1980-6574 - está licenciada sob Creative Commons - Atribuição 3.0 